\title{
Factors Associated with Non-Remission in Bipolar Disorder: The Multicenter Treatment Survey for Bipolar Disorder in Psychiatric Outpatient Clinics (MUSUBI)
}

This article was published in the following Dove Press journal:

Neuropsychiatric Disease and Treatment

Takashi Tsuboi, (iD) ${ }^{1,2}$

Takefumi Suzuki, (iD) 2,3

Takaharu Azekawa, ${ }^{4}$

Naoto Adachi, ${ }^{4}$ Hitoshi Ueda, ${ }^{4}$

Kouji Edagawa, 4

Eiichi Katsumoto, ${ }^{4}$

Yukihisa Kubota, (iD) ${ }^{4}$

Eiichiro Goto, ${ }^{4}$ Seiji Hongo, ${ }^{4}$

Yoichiro Watanabe, ${ }^{4}$

Masaki Kato, (DD ${ }^{2,5}$ Norio

Yasui-Furukori, ${ }^{2,6}$

Reiji Yoshimura, ${ }^{2,7}$

Atsuo Nakagawa, (iD ${ }^{2,8}$

Toshiaki Kikuchi, 2,8

Koichiro Watanabe ${ }^{1,2}$

'Department of Neuropsychiatry, Kyorin University School of Medicine, Tokyo, Japan;

${ }^{2}$ The Japanese Society of Clinical

Neuropsychopharmacology, Tokyo, Japan;

${ }^{3}$ Department of Neuropsychiatry, University of Yamanashi Faculty of Medicine, Yamanashi, Japan; ${ }^{4}$ The Japanese Association of NeuroPsychiatric Clinics, Tokyo, Japan;

${ }^{5}$ Department of Neuropsychiatry, Kansai

Medical University, Osaka, Japan;

${ }^{6}$ Department of Psychiatry, Dokkyo Medical University, Tochigi, Japan; ${ }^{7}$ Department of Psychiatry, University of Occupational and Environmental Health, Fukuoka, Japan;

${ }^{8}$ Department of Neuropsychiatry, Keio University School of Medicine, Tokyo, Japan

Correspondence: Takashi Tsuboi Department of Neuropsychiatry, Kyorin University School of Medicine, 6-20-2,

Shinkawa, Mitaka-Shi, Tokyo I8I-86II, Japan

Tel +8I $4224755 \mathrm{II}$

$\mathrm{Fax}+81422454697$

Email takashi.tsuboi.082I@gmail.com
Purpose: The aim of this study was to identify factors associated with non-remission in bipolar disorder.

Patients and Methods: The multicenter treatment survey for bipolar disorder in psychiatric outpatient clinics (MUSUBI) study used a questionnaire administered at 176 clinics throughout Japan from September to October 2016. Clinic psychiatrists performed a retrospective medical record survey of consecutive cases with bipolar disorder. Patients were considered to be in remission if they met all of the following criteria: they were not in a mixed state, their manic or depressive symptoms were either borderline or nonexistent (corresponding to 2 or 1 points on the Clinical Global Impressions Scale, Bipolar Version), and their psychiatrists clinically considered them to be in remission. Enrolled patients were classified into remitters group and non-remitters group and demographic and clinical characteristics were contrasted between the groups. Non-remitters were compared with remitters, using a series of logistic regression analyses.

Results: A total of 3130 patients (1420 men; mean age: 50.3 years) were included in this study; 1307 patients $(41.8 \%)$ were in remission. Of the remaining 1823 patients, 1260 $(40.3 \%)$ had mild to severe depression, 261 (8.3\%) suffered from manic or hypomanic episodes, and $302(9.6 \%)$ were in a mixed state. Logistic regression analyses found the following eight factors to be significantly correlated with non-remission in patients with bipolar disorder: female gender, younger age, unemployed status, rapid cycling pattern, comorbid alcohol/substance abuse, poorer social function, lithium non-use, and antidepressant use.

Conclusion: The MUSUBI study, the largest nationwide investigation on bipolar disorder, identified eight clinically relevant factors associated with non-remission in bipolar patients. They have important clinical implications; further prospective studies are necessary to replicate these findings and to guide better managements for those in serious needs.

Keywords: bipolar disorder, non-remission, nationwide study, mood stabilizer, antipsychotics

\section{Introduction}

Bipolar disorder is frequently a severe, recurrent, and debilitating psychiatric disorder. ${ }^{1}$ Although several treatment-guidelines and meta-analyses on bipolar disorder have been published across the globe, ${ }^{2-7}$ the problem of non-response and non-remission has been ubiquitous in the real-world clinical settings. As such, 
many individuals with bipolar disorders still suffer from troublesome symptoms, resulting in substantial financial, social, or work-related issues. ${ }^{8}$

Bipolar disorder is classified into bipolar I disorder, bipolar II disorder, and bipolar disorder not otherwise specified, for which different psychopharmacological treatment is recommended. In addition, the first-line medications are divided individually for acute mania phase, bipolar depression phase, and maintenance phase. Such a complexity constitutes the hurdles in conducting clinical research on bipolar disorders in a longitudinal manner. Only a few large-scale studies are available to guide clinical management (e.g., the Systematic Treatment Enhancement Program for Bipolar Disorder (STEP-BD) ${ }^{9}$ ), and maintenance treatment is complicated in that the studies usually adopt an enrichment design; ${ }^{10}$ information is critically racking on what to do to who fail to show response or remission with standard treatments.

On the other hand, it is frequently challenging to distinguish bipolar disorder from unipolar depression; the average delay in diagnosis of bipolar disorder is reported to be $5-10$ years after the symptoms begin. ${ }^{11,12}$ Even after an accurate diagnosis is made, it still remains a challenge to achieve complete remission of all symptoms with the currently available psychotropic medications, and symptoms may even become progressively more severe over time. ${ }^{13-15}$ It is thus of high clinical importance to extract factors associated with remission of bipolar disorder. According to the European Mania in Bipolar Longitudinal Evaluation of Medication (EMBLEM) study, patients who had a higher overall score on the Clinical Global Impression Scale, Bipolar Version for bipolar disorder (CGI-BP), who had depressive episodes in the year before, who had poor social functioning, and who were treated with typical antipsychotics or antidepressants were found to be less likely to achieve remission or recovery. ${ }^{16}$ However, while treatment response has usually expressed with a "relative" threshold (e.g., 50\% decrease in the representative rating scales), the definition of remission in patients with bipolar disorder, albeit often arbitrarily expressed with an absolute threshold (e.g., a score of less than 7 on the Montgomery-Asberg depression rating scale $^{18}$ ), has not been unequivocally established to date. ${ }^{17}$

With these backgrounds, the goal of this study was to perform a large-scale investigation of patients with bipolar disorder in order to identify factors associated with nonremission.

\section{Materials and Methods}

\section{Study Design and Subjects}

The multicenter treatment survey for bipolar disorder in psychiatric outpatient clinics (MUSUBI) is a crosssectional study in which a questionnaire was administered at 176 clinics located across Japan from September to October 2016. Patients diagnosed with bipolar disorder according to the Tenth Revision of the International Classification of Diseases and Related Health Problems, 10th edition (ICD-10) and treated at the aforementioned clinics were included; patients with serious physical conditions such as terminal cancer or intractable diseases were excluded. Almost all psychiatrists involved in this study were psychiatric specialists certified by the Japanese Society of Psychiatry and Neurology, and/or designated psychiatrists certified by the Japanese Ministry of Health, Labour and Welfare.

\section{Study Procedures}

Clinic psychiatrists were asked to complete a questionnaire by performing a retrospective medical record survey of consecutive cases with bipolar disorder, beginning with the first patient who visited the clinic during the study period. The questionnaire included patient characteristics (age, gender, height, weight, academic background, and occupational status), comorbidities, psychiatric symptoms, course of the illness, the Global Assessment of Functioning (GAF) score, and details of pharmacological treatment. We mailed this questionnaire to each outpatient clinic in 20 copies and analyzed the responses.

This study focused on factors associated with remission (versus non-remission). Based on the proposal of the International Society of Bipolar Disorders, this study defined patients as being in remission if they met all three of the following criteria: they were not in a mixed state, their manic or depressive symptoms were either borderline or nonexistent (corresponding to 2 or 1 points on the CGI-BP), ${ }^{17}$ and their psychiatrists clinically considered them to be in remission. Non-remitted patients comprised all the rest of bipolar patients and might have been in depressive, (hypo)manic and mixed state; we hereby term them remitters and nonremitters, respectively.

\section{Statistical Analysis}

SPSS version 25 (SPSS, IBM Corp, Armonk, NY, USA) was used for the analyses. All of the statistical tests were based on a two-sided significance level of 0.05 unless 
otherwise specified. Demographic and clinical characteristics were analyzed using Mann-Whitney $U$-test or Kruskal-Wallis test for differences between remitters and non-remitters. For pharmacological treatment, comparisons were made among the following four groups: remission group, manic state group, depressive state group and mixed state group. Pearson's chi-square test was first used to compare the four groups, and then drugs with significant differences were analyzed using Mann-Whitney $U$-test for each pair of groups. A univariate logistic regression analysis was first performed to identify the demographic and clinical features of non-remitters. A multivariate logistic regression analysis was then performed for the features with a p-value of less than 0.001 in univariate analysis. The factors with a $p$ value of 0.05 or less in the multivariate analysis were extracted as being significantly associated with non-remission.

\section{Ethics}

This study was conducted in accordance with the Declaration of Helsinki and the Japanese Ethical Guidelines for Epidemiological Research. Prior to the initiation of the study, the study protocol was reviewed and approved by the institutional review board of the ethics committee of the Japanese Association of Neuro-Psychiatric Clinics (ID:
20160822). Since this was a retrospective medical record survey, informed consent was exempted but we instead released information on this research so that patients were free to opt out. To protect patients' personal information, a study-specific unique subject identification number was assigned to each patient for linkable anonymity.

\section{Results}

\section{Demographic Characteristics}

Completed questionnaires on 3213 patients with bipolar disorder were returned from 176 outpatient facilities participating in this study; response rate was $91.3 \%$. Eightythree patients, who missed much of the data on psychiatric symptoms or medications, were excluded, leaving a total of 3130 patients to be included in this study.

The demographic characteristics of the study sample are summarized in Table 1. The mean patient age was 50.3 years, and $45.4 \%$ of the patients were men. As for occupational status, $35.3 \%$ of the patients were working full time while $33.7 \%$ were unemployed. Regarding educational background, $37.1 \%$ of the patients were university graduates. The average body mass index was $23.4 \mathrm{~kg} / \mathrm{m}^{2}$. There were statistically significant differences between remitters vs non-remitters in terms of gender, age, and current work status.

Table I Demographic Characteristics

\begin{tabular}{|c|c|c|c|c|}
\hline Variables & Total & Non-Remitters & Remitters & $\mathrm{p}$ value \\
\hline $\mathrm{N}$ & 3130 & 1823 & 1307 & \\
\hline Male gender $\mathrm{n}(\%)$ & $1420(45.4)$ & $763(4 I .9)$ & $657(50.3)$ & $<0.001$ \\
\hline Age (years): mean $\pm S D$ & $50.3 \pm 13.9$ & $48.9 \pm 13.3$ & $52.2 \pm 14.4$ & $<0.001$ \\
\hline Current work status: n (\%) & & & & $<0.001$ \\
\hline Unemployed & $1054(33.7)$ & $690(37.8)$ & $364(27.9)$ & \\
\hline Full-time manager & $186(5.9)$ & $88(4.8)$ & $98(7.5)$ & \\
\hline Full-time worker & $918(29.3)$ & $457(25.1)$ & $461(35.3)$ & \\
\hline Part-time worker & $294(9.4)$ & $165(9.1)$ & $129(9.9)$ & \\
\hline Housewife & $497(15.9)$ & $305(16.7)$ & $192(14.7)$ & \\
\hline Student & $34(1.1)$ & $20(1.1)$ & $14(1.1)$ & \\
\hline Employment of the disabled & $90(2.9)$ & $65(3.6)$ & $25(1.9)$ & \\
\hline Educational background: n (\%) & & & & 0.082 \\
\hline Special support education school & $10(0.3)$ & $5(0.3)$ & $5(0.4)$ & \\
\hline Junior high school & 146 (4.7) & $85(4.7)$ & $61(4.7)$ & \\
\hline High school, vocational school & $1394(44.5)$ & $844(46.3)$ & $550(42.1)$ & \\
\hline Junior college, technical college & $271(8.7)$ & $173(9.5)$ & $98(7.5)$ & \\
\hline University & $1059(33.8)$ & $588(32.3)$ & $47 \mid(36.0)$ & \\
\hline Master's degree or higher & $102(3.3)$ & $62(3.4)$ & $40(3.1)$ & \\
\hline Body Mass Index $\left(\mathrm{kg} / \mathrm{m}^{2}\right):$ mean $\pm S D$ & $23.4 \pm 3.9$ & $23.4 \pm 3.9$ & $23.4 \pm 3.8$ & 0.681 \\
\hline
\end{tabular}




\section{Clinical Characteristics}

There were 1307 patients $(41.8 \%)$ in remission on a crosssectional basis. Of the remaining 1823 patients who comprised the non-remission group, 1260 (40.3\%) had mild to severe depression, $261(8.3 \%)$ suffered from manic or hypomanic episodes with a score in the CGI-BP of 3 or more, and $302(9.6 \%)$ were in a mixed state. A total of 329 patients $(10.5 \%)$ had bipolar disorder with a rapid cycling characteristic.

The clinical characteristics of the patients are summarized in Table 2. The mean age at the onset of the first episode was 34.7 years. Our sample included 610 patients $(19.5 \%)$ with coexisting psychiatric disorders, $31.0 \%$ with physical complications, and 5.3\% with alcohol/substance abuse. There were statistically significant differences between the groups in terms of age of onset, comorbidity of personality disorders, comorbidity of pervasive developmental disorders, rapid cycling pattern, comorbidity of alcohol/substance abuse, and social function. Lithium use was more frequent among remitters but psychopharmacological treatment was more extensive among nonremitters.

\section{Pharmacological Treatment}

Almost all of the patients had been receiving any psychopharmacological treatment. The results, in relation to mood states, are detailed in Table 3. Many patients across the four groups were treated with lithium. Lithium was used significantly more frequently in the remission group than in the depressive state group $(53.9 \%$ vs $41.5 \%$, respectively; $\mathrm{P}<0.001$ ). On the other hand, lamotrigine was administered significantly more frequently in the depressive state group and the mixed group than in the remission group $(28.6 \%$ and $23.2 \%$ vs $20.1 \%$, respectively; $\mathrm{P}<0.001$ and $\mathrm{P}=0.017$ ). While more than 20 antipsychotic drugs were available in Japan at the time of this survey, Table 3 lists only representative ones. Aripiprazole was the most commonly prescribed antipsychotic, and was administered frequently in the depressive state group and the mixed state in particular. Furthermore, a total of 1291 patients $(41.2 \%)$ were receiving various types of antidepressant drugs; 155 patients $(5.0 \%)$ were treated with tricyclic antidepressants and 120 patients (3.8\%) were treated only with antidepressants in the absence of mood stabilizers or antipsychotics. Of the 1260 patients in the

Table 2 Comparison of Remitters vs Non-Remittors

\begin{tabular}{|c|c|c|c|c|}
\hline Variables & Total & Non-Remitters & Remitters & p value \\
\hline $\mathrm{N}$ & 3130 & 1823 & 1307 & \\
\hline Age of onset (years): mean $\pm S D$ & $34.7 \pm 12.5$ & $33.5 \pm 12.0$ & $36.2 \pm 13.0$ & $<0.001$ \\
\hline Personality disorders: $\mathrm{n}(\%)$ & $|8|(5.8)$ & $148(8.1)$ & $32(2.4)$ & $<0.001$ \\
\hline Pervasive developmental disorders: $\mathrm{n}$ (\%) & $207(6.6)$ & $148(8.1)$ & $59(4.5)$ & $<0.001$ \\
\hline Alcohol/substance abuse: n (\%) & $167(5.3)$ & $133(7.3)$ & $34(2.6)$ & $<0.001$ \\
\hline Physical comorbidity: n (\%) & $968(30.9)$ & $557(30.6)$ & $4 I I(3 I .4)$ & 0.601 \\
\hline Rapid cycling: $n$ (\%) & $329(10.5)$ & $264(14.5)$ & $65(5.0)$ & $<0.001$ \\
\hline Social function: $\mathrm{n}(\%)$ & & & & $<0.001$ \\
\hline Global Assessment of Functioning $8 \mathrm{I}-100$ & $1008(32.2)$ & $203(I I . I)$ & $805(61.6)$ & \\
\hline Global Assessment of Functioning $6 \mathrm{I}-80$ & $1407(45.0)$ & $972(53.3)$ & $435(33.3)$ & \\
\hline Global Assessment of Functioning $4 \mathrm{I}-60$ & $606(19.4)$ & $550(30.2)$ & $56(4.3)$ & \\
\hline Global Assessment of Functioning $\mathrm{I}-40$ & $87(2.8)$ & $81(4.4)$ & $6(0.5)$ & \\
\hline Pharmacological treatment: $\mathrm{n}(\%)$ & $3104(99.2)$ & $|8| \mid(99.3)$ & $1293(98.9)$ & 0.147 \\
\hline \multicolumn{5}{|l|}{ Mood stabilizers: $n$ (\%) } \\
\hline Lithium & I $488(47.5)$ & $783(43.0)$ & 705 (53.9) & $<0.001$ \\
\hline Valproic acid & $893(28.5)$ & $533(29.2)$ & $358(27.4)$ & 0.259 \\
\hline Carbamazepine & $149(4.8)$ & $97(5.3)$ & $52(4.0)$ & 0.082 \\
\hline Lamotrigine & $744(23.8)$ & $48 I(26.4)$ & $263(20.1)$ & $<0.001$ \\
\hline Antipsychotics: $n(\%)$ & $1692(54.1)$ & $108 \mid(59.3)$ & $611(46.7)$ & $<0.001$ \\
\hline Antidepressants: $\mathrm{n}(\%)$ & $|29|(4 \mid .2)$ & $828(45.4)$ & $463(35.4)$ & $<0.001$ \\
\hline Anxiolytics: n (\%) & $1133(36.2)$ & $739(40.6)$ & $394(30.2)$ & $<0.001$ \\
\hline Hypnotics: n (\%) & $1886(60.3)$ & $1213(66.5)$ & $673(5 \mathrm{I} .5)$ & $<0.001$ \\
\hline
\end{tabular}


Table 3 Psychotropics According to Mood State

\begin{tabular}{|c|c|c|c|c|c|c|c|}
\hline & Remission (Re) & Manic State (Ma) & Depressive State (De) & Mixed State (Mi) & $p$ value & & \\
\hline$n$ & 1307 & 261 & 1260 & 302 & Re vs Ma & Re vs, De & Re vs Mi \\
\hline \multicolumn{8}{|l|}{ Mood stabilizers: n (\%) } \\
\hline Lithium & 705 (53.9\%) & $134(51.3 \%)$ & $523(41.5 \%)$ & $126(41.7 \%)$ & 0.442 & $<0.001$ & 0.083 \\
\hline Valproic acid & $358(27.4 \%)$ & 89 (34.1\%) & 339 (26.9\%) & 105 (34.8\%) & 0.028 & 0.448 & $<0.001$ \\
\hline Carbamazepine & 52 (4.0\%) & $20(9.3 \%)$ & $58(4.6 \%)$ & 19 (6.3\%) & 0.009 & 0.54 & 0.02 \\
\hline Lamotrigine & $263(20.1 \%)$ & 51 (19.5\%) & $360(28.6 \%)$ & $70(23.2 \%)$ & 0.83 & $<0.001$ & 0.017 \\
\hline \multicolumn{8}{|l|}{ Antipsychotics: n (\%) } \\
\hline Aripiprazole & 194 (14.8\%) & 38 (I4.6\%) & $314(24.9 \%)$ & 60 (19.9\%) & 0.906 & $<0.001$ & 0.001 \\
\hline Olanzapine & $150(11.5 \%)$ & 35 (I3.4\%) & $188(14.9 \%)$ & $46(15.2 \%)$ & 0.377 & 0.023 & 0.007 \\
\hline Quetiapine & $136(10.4 \%)$ & 38 (14.6\%) & 155 (12.3\%) & 45 (14.9\%) & 0.051 & 0.219 & 0.002 \\
\hline Risperidone & 49 (3.7\%) & $13(5.0 \%)$ & 50 (4.0\%) & II (3.6\%) & 0.351 & 0.897 & 0.729 \\
\hline
\end{tabular}

depressive state group, $659(52.3 \%)$ were receiving antidepressant treatment, $124(9.8 \%)$ were treated with tricyclic antidepressants, and $59 \quad(4.7 \%)$ received only antidepressants.

\section{Factors Associated with Non-Remission}

After the patients were classified into remitters and nonremitters, a univariate logistic regression analysis was performed on all demographic and clinical characteristics. Of these, 15 with a p-value of less than 0.001 were identified as candidate factors that were correlated with non-remission.

A multivariate logistic regression analysis was then performed on the following 15 factors: female, younger age, unemployed status, earlier age of onset, comorbidity of personality disorders, comorbidity of pervasive developmental disorders, rapid cycling pattern, comorbidity of alcohol/substance abuse, poorer social function, lithium non-use, lamotrigine use, antipsychotic use, antidepressant use, anxiolytic use, and hypnotic use. As shown in Table 4, the following eight factors were found to be significantly correlated with non-remission in Japanese outpatients with bipolar disorder: female gender, younger age, unemployed status, rapid cycling pattern, comorbidity of alcohol/substance abuse, poorer social function, lithium non-use, and antidepressant use.

\section{Discussion}

This is the first multicenter nationwide study, to the best of our knowledge, an effort to characterize the current status on treatment of bipolar disorder in Japan. The strength of the study is that as many as 3130 outpatients with bipolar disorder were included and, along with the STEP-BD, it is one of the largest clinical studies on this illness. The participants in this study were more likely to have graduated from university than the general population in Japan, and a similar trend was seen in the STEP-BD sample vs the US general population. ${ }^{18}$ MacCabe et al suggested that bipolar disorder may be associated with a high intelligence quotient or excellent school performance, ${ }^{19}$ and the results may support such theory. Furthermore, both this study and the STEP-BD study showed that the percentage of full-time workers was lower than the general population, and conversely the percentage of unemployed people was higher. ${ }^{18}$ According to Kessler et al, a greater employment loss

Table 4 Factors Associated with Non-Remission

\begin{tabular}{|c|c|c|c|}
\hline Candidate Factors & p value & $\begin{array}{l}\text { Odds } \\
\text { Ratio }\end{array}$ & $\begin{array}{l}\text { 95\% Confidence } \\
\text { Interval }\end{array}$ \\
\hline Female gender & 0.001 & 1.364 & $1.137-1.637$ \\
\hline Younger age & 0.028 & 0.99 & $0.981-0.999$ \\
\hline Unemployed & $<0.001$ & 1.592 & $1.283-1.975$ \\
\hline Earlier onset & 0.591 & 1.003 & $0.993-1.012$ \\
\hline Personality disorders & 0.295 & 1.283 & $0.804-2.047$ \\
\hline $\begin{array}{l}\text { Pervasive } \\
\text { developmental } \\
\text { disorders }\end{array}$ & 0.291 & 0.817 & $0.561-1.189$ \\
\hline Rapid cycling & 0.008 & 1.556 & $1.12-2.162$ \\
\hline $\begin{array}{l}\text { Alcohol/substance } \\
\text { abuse }\end{array}$ & $<0.001$ & 2.221 & $1.432-3.445$ \\
\hline Poorer social function & $<0.001$ & 6.949 & $5.887-8.204$ \\
\hline Lithium non-use & $<0.001$ & 1.488 & $1.235-1.793$ \\
\hline Lamotrigine & 0.227 & $\mathrm{I} .144$ & $0.92-1.424$ \\
\hline Antipsychotics & 0.594 & 0.951 & $0.789-1.145$ \\
\hline Antidepressants & 0.02 & 1.25 & $|.035-1.5|$ \\
\hline Anxiolytics & 0.279 & 1.157 & $0.888-1.508$ \\
\hline Hypnotics & 0.093 & 1.263 & $0.96 I-1.66$ \\
\hline
\end{tabular}


was noted in bipolar disorder than in major depressive disorder. ${ }^{20}$ In addition, their study showed that more severe and persistent depressive episodes in bipolar disorder were associated with employment disadvantage, ${ }^{20}$ which is consistent with the results of a meta-analysis that indicated the course of illness affected work status, irrespective of years of education. $^{21}$

Concerning the cross-sectional prevalence of each phase of bipolar disorder in this study, about $40 \%$ of the patients were in remission or a depressive state, while fewer than $10 \%$ were in a manic or mixed state. Judd et al investigated the long-term natural history of the symptomatic status of bipolar I and II disorders, and found that in these two conditions, depressive (31.9\% and $50.3 \%$, respectively) and euthymic ( $52.9 \%$ and $46.1 \%$, respectively) symptoms were much more dominant than manic/hypomanic $(9.3 \%$ and $1.3 \%$, respectively) and cycling/mixed (5.9\% and $2.3 \%$, respectively) symptoms. ${ }^{22,23}$ The proportions of the disease states in our 3130 patients were generally consistent with the previous research, which might guarantee generalizability of study results although this study failed to distinguish bipolar subtypes.

Lithium was found to be the most commonly utilized drug in all phases of bipolar disorder, which is consistent with the recommendations of the Japanese guidelines. ${ }^{24}$ According to the evidence-based Canadian Network for Mood and Anxiety Treatments (CANMAT) and the International Society for Bipolar Disorders (ISBD) 2018 guidelines for the management of patients with bipolar disorder, lithium has a level 1 recommendation for treatment of acute mania and the maintenance phase, and a level 2 recommendation for treatment of acute depression. ${ }^{2}$ This reflects the notion that the effectiveness of lithium is well established as an initial treatment that usually continues to the maintenance phase of the illness in bipolar disorder. ${ }^{25}$ Further, lithium may be effective for patients with mixed state although the evidence is currently insufficient. ${ }^{26}$ Although $41.7 \%$ of mixed state patients were treated with lithium in this study, there might have been in more remission cases should more patients would have been treated with lithium. Lamotrigine was the next most frequently prescribed drug in the depressive phase in this study. Lamotrigine is recommended for acute depression by the JSMD 2017 and CANMAT/ISBD 2018 guidelines, ${ }^{2}$ but a recent multiple-treatments meta-analysis of bipolar depression does not endorse the drug due to its high risk of switching and less robust efficacy. ${ }^{7}$ Like lithium, quetiapine is recommended by the guidelines for all phases, ${ }^{2,3}$ but it was used infrequently in this study. This may be because quetiapine was off-label for bipolar disorder at the time of this survey and is contraindicated for those with diabetes mellitus in Japan. Meanwhile, aripiprazole was the most frequently utilized antipsychotic drug despite the fact that most guidelines on bipolar disorder do not recommend it for acute depression..$^{2-4,24}$ This may be because it is infrequently difficult to distinguish between bipolar depression and unipolar depression; ${ }^{11,12}$ aripiprazole is the only antipsychotic agent that is covered by insurance for unipolar depression in Japan. Finally, both antidepressant monotherapy prescription and tricyclic antidepressant use were relatively infrequent, which is reassuring and consistent with the guidelines. ${ }^{2-4,24}$ To interpret the results more precisely, however, it is important to be aware of the fact that there are many types of antidepressants and antipsychotics available, and a combination therapy within each category or across categories is also often clinically adopted.

Bipolar disorder is a highly recurrent disease; $70-90 \%$ of patients are reported to relapse within 5 years. ${ }^{1}$ Alternatively, it is of high clinical relevance to identify factors associated with remission. However, clinical trials of maintenance therapy for bipolar disorder are usually short for this often chronic condition, are not necessarily consistent in methodology and may be somewhat biased through an enrichment design. In the World Federation of Societies of Biological Psychiatry (WFSBP) guidelines for the biological treatment of bipolar disorders, it was pointed out that clinical studies on the long-term treatment of bipolar disorder have used various primary outcomes. ${ }^{4}$ A number of other factors have been inconsistent as well, such as inclusion criteria, observation periods, and definitions of remission and recovery, all making it difficult to draw firm conclusions. ${ }^{4,27}$ Thus we believe that although this study consisted of a retrospective chart review, the results have important clinical implications. A naturalistic Taiwanese study found that comorbid substance use disorders, earlier age at onset, and greater manic symptom severity were statistically significant predictors of a longer time to achieve remission in bipolar mania. ${ }^{28}$ Another prospective 5-year follow-up study from Denmark found that non-remission in bipolar disorder increased with younger age, co-morbid anxiety, and suicidal ideation. ${ }^{29}$ While both of the previous studies included hundreds of patients, a strength of this work is that it explored factors associated with remission status in over 3000 outpatients with bipolar disorder. The eight factors we found in this study have substantial clinical relevance in a quest for remission status in bipolar patients and the findings are expected to be incremental to the existing literature on 
bipolar disorder in the Asian region. An important finding is that non-use of lithium was associated with nonremission. Lithium non-use might be a result of excessive fear of its side effects. Clinicians are required to well balance the risks and benefits of lithium treatment in bipolar disorder.

There are several limitations on this study that should be acknowledged to better interpret the results. First, since this was a retrospective chart study, the identified factors associated with non-remission in bipolar disorder may not necessarily establish causation. This would be considered as the most outstanding limitation of this work. Welldesigned prospective studies are necessary to identify factors that are causally related to remission status. Second, it is possible that missing information on the clinical variables limited the analysis. However, only 83 cases (out of 3213 patients: $2.6 \%$ ) were excluded because of missing data. Third, the characteristics of this study may limit generalizability to other populations. This study of the Japanese outpatients with bipolar disorder included slightly more females than males, but the incidence of bipolar disorder has been reported to be equal not only between genders but also across different cultural and ethnic groups. ${ }^{30,31}$ Moreover, the average age of onset of mood disturbance was 34.7 y.o. in this study, whereas it was 17.4 y.o. in the STEP-BD study. ${ }^{18}$ Fourth, in this study, remission was arbitrarily based on the impression of the evaluators; it would have been more precisely defined with assessment scales such as the Hamilton Depression Rating Scale or the MADRS and the Young Mania Rating Scale (YMRS). ${ }^{17}$ But it would contrarily have been impractical for clinic psychiatrists to perform these assessment scales in daily clinical practice. Fifth, due to lack of data that were not included in the survey, this study failed to analyze several factors identified in previous studies as potentially affecting remission status, such as the number of previous episodes, impulsivity, adherence and time to respond to treatment drugs. ${ }^{32-35}$ Sixth, this study did not distinguish bipolar I disorder from bipolar II disorder and the dose of medications was not considered. Seventh, neuromodulation treatments such as electroconvulsive therapy (ECT) and transcranial magnetic stimulation (TMS) may be effective for refractory bipolar disorder. ${ }^{36,37}$ However, ECT is a treatment performed in a hospital in Japan; this study was conducted at outpatient psychiatric clinics. In addition, TMS is not approved for bipolar disorder in Japan. Finally, as is always problematic for this kind of retrospective chart reviews, there are many possible biases and unmeasured confounders.

\section{Conclusion}

To the best of our knowledge, this survey is the largest nationwide investigation thus far on the remission status of patients with bipolar disorder conducted in Japan. Data from more than 3000 outpatients indicated that in real-world clinical settings, not all treatment selections conform to bipolar disorder treatment guidelines. Most importantly, this study suggests that the following eight factors are associated with non-remission in patients with bipolar disorder: female gender, younger age, unemployed status, rapid cycling pattern, comorbidity of alcohol/substance abuse, poorer social function, non-use of lithium, and antidepressant use. They have important clinical implications and further prospective interventions as well as observational studies are necessary to replicate these findings in order to inform better managements for those in serious needs.

\section{Consent for Publication}

Since this study was a retrospective medical record survey, informed consent was exempted but we instead released information on this research so that patients were free to opt out.

\section{Data Sharing Statement}

The datasets of the current study are available from the corresponding author on reasonable request.

\section{Ethics and Consent Statement}

This study protocol was reviewed and approved by the institutional review board of the ethics committee of the Japanese Association of Neuro-Psychiatric Clinics (ID: 20160822).

\section{Acknowledgments}

A part of this study was presented at 32nd European College of Neuropsychopharmacology Congress, which has been published in European Neuropsychopharmacology, Volume 29, Supplement 6, Pages S1-S598, only as an abstract form. The authors thank the following psychiatrists belonging to the Japanese Association of Neuro-Psychiatric Clinics: Dr. Kazuhira Miki, Dr. Toshihiko Lee, Dr. Norio Okamoto, Dr. Makoto Nakamura, Dr. Junkou Sato, Dr. Kazunori Otaka, Dr. Satoshi Terada, Dr. Tadashi Ito, Dr. Munehide Tani, Dr. Atsushi Satomura, Dr. Hiroshi Sato, Dr. Hideki Nakano, Dr. Yoichi Nakaniwa, Dr. Eiichi Hirayama, 
Dr. Keiichi Kobatake, Dr. Koji Tanaka, Dr. Mariko Watanabe, Dr. Shiguyuki Uehata, Dr. Asana Yuki, Dr. Nobuko Akagaki, Dr. Michie Sakano, Dr. Akira Matsukubo, Dr. Yukihisa Kibota, Dr. Yasuyuki Inada, Dr. Hiroshi Oyu, Dr. Tsuneo Tsubaki, Dr. Tatsuji Tamura, Dr. Shigeki Akiu, Dr. Atsuhiro Kikuchi, Dr. Keiji Sato, Dr. Kazuyuki Fujita, Dr. Fumio Handa, Dr. Hiroyuki Karasawa, Dr. Kazuhiro Nakano, Dr. Kazuhiro Omori, Dr. Seiji Tagawa, Dr. Daisuke Maruno, Dr. Hiroaki Furui, Dr. You Suzuki, Dr. Takeshi Fujita, Dr. Yukimitsu Hoshino, Dr. Kikuko Ota, Dr. Takaharu Azekawa, Dr. Akira Itami, Dr. Kenichi Goto, Dr. Yoshiaki Yamano, Dr. Kiichiro Koshimune, Dr. Junko Matsushita, Dr. Takatsugu Nakayama, Dr. Kazuyoshi Takamuki, Dr. Nobumichi Sakamoto, Dr. Eiichi Katsumoto, Dr. Miho Shimizu, Dr. Muneo Shimura, Dr. Norio Kawase, Dr. Ryouhei Takeda, Dr. Takuya Hirota, Dr. Hideko Fujii, Dr. Yoichiro Watanabe, Dr. Riichiro Narabayashi, Dr. Yutaka Fujiwara, Dr. Kazu Kobayashi, Dr. Yuko Urabe, Dr. Miyako Oguru, Dr. Osamu Miura, Dr. Yoshio Ikeda, Dr. Hitoshi Ueda, Dr. Hidemi Sakamoto, Dr. Yosuke Yonezawa, Dr. Yoichi Takei, Dr. Toshimasa Sakane, Dr. Kiyoshi Oka, Dr. Kyoko Tsuda, Dr. Shigemitsu Hayashi, Dr. Kunihiko Kawamura, Dr. Yasushi Furuta, Dr. Kazuko Miyauchi, Dr. Yoshio Miyauchi, Dr. Mikako Oyama, Dr. Keizo Hara, Dr. Misako Sakamoto, Dr. Shigeki Masumoto, Dr. Yasuhiro Kaneda, Dr. Yoshiko Kanbe, Dr. Masayuki Iwai, Dr. Naohisa Waseda, Dr. Nobuhiko Ota, Dr. Takahiro Hiroe, Dr. Ippei Ishii, Dr. Hideki Koyama, Dr. Terunobu Otani, Dr. Osamu Takatsu, Dr. Takashi Ito, Dr. Norihiro Marui, Dr. Toru Takahashi, Dr. Tetsuro Oomori, Dr. Toshihiko Fukuchi, Dr. Kazumichi Egashira, Dr. Kiyoshi Kaminishi, Dr. Ryuichi Iwata, Dr. Satoshi Kawaguchi, Dr. Yoshinori Morimoto, Dr. Hirohisa Endo, Dr. Yasuo Imai, Dr. Eri Kohno, Dr. Aki Yamamoto, Dr. Naomi Hasegawa, Dr. Sadamu Toki, Dr. Hideyo Yamada, Dr. Hiroyuki Taguchi, Dr. Hiroshi Yamaguchi, Dr. Hiroki Ishikawa, Dr. Sakura Abe, Dr. Kazuhiro Uenoyama, Dr. Kazunori Koike, Dr. Yoshiko Kamekawa, Dr. Michihito Matsushima, Dr. Ken Ueki, Dr. Sintaro Watanabe, Dr. Tomohide Igata, Dr. Yoshiaki Higashitani, Dr. Eiichi Kitamura, Dr. Junko Sanada, Dr. Takanobu Sasaki, Dr. Kazuko Eto, Dr. Ichiro Nasu, Dr. Kenichiro Sinkawa, Dr. Yukio Oga, Dr. Michio Tabuchi, Dr. Daisuke Tsujimura, Dr. Tokunai Kataoka, Dr. Kyohei Noda, Dr. Nobuhiko Imato, Dr. Ikuko Nitta, Dr. Yoshihiro Maruta, Dr. Satoshi Seura, Dr. Toru Okumura, Dr. Osamu Kino, Dr. Tomoko Ito, Dr. Ryuichi Iwata, Dr. Wataru Konno, Dr. Toshio Nakahara, Dr. Masao
Nakahara, Dr. Hiroshi Yamamura, Dr. Masatoshi Teraoka, Dr. Eiichiro Goto, Dr. Masato Nishio, Dr. Koji Edagawa, Dr. Miwa Mochizuki, Dr. Tsuneo Saitoh, Dr. Tetsuharu Kikuchi, Dr. Chika Higa, Dr. Hiroshi Sasa, Dr. Yuichi Inoue, Dr. Muneyoshi Yamada, Dr. Yoko Fujioka, Dr. Kuniaki Maekubo, Dr. Hiroaki Jitsuiki, Dr. Toshihito Tsutsumi, Dr. Yasumasa Asanobu, Dr. Seiji Inomata, Dr. Kazuhiro Kodama, Dr. Aikihiro Takai, Dr. Asako Sanae, Dr. Shinichiro Sakurai, Dr. Kazuhide Tanaka, Dr. Masahiko Shido, Dr. Haruhisa Ono, Dr. Wataru Miura, Dr. Yukari Horie, Dr. Tetso Tashiro, Dr. Tomohide Mizuno, Dr. Naohiro Fujikawa, Dr. Hiroshi Terada, Dr. Kenji Taki, Dr. Kyoko Kyotani, Dr. Masataka Hatakoshi, Dr. Katsumi Ikeshita, Dr. Keiji Kaneta, Dr. Ritsu Shikiba, Dr. Tsuyoshi Iijima, Dr. Masaru Yoshimura, Dr. Naoto Adachi, Dr. Masumi Ito, Dr. Shunsuke Murata, Dr. Seiji Hongo, Dr. Mio Mori, and Dr. Toshio Yokouchi.

\section{Author Contributions}

All authors designed this study. Dr. Tsuboi analyzed the data and wrote the first draft of the manuscript. All authors contributed to data analysis, drafting or revising the article, gave final approval of the version to be published, and agree to be accountable for all aspects of the work.

\section{Funding}

This study was supported by Ken Tanaka memorial research grant.

\section{Disclosure}

Dr. Tsuboi has received consultant fees from Pfizer and speaker's honoraria from Eli Lilly, Meiji-Seika Pharma, MSD, Janssen Pharmaceutical, Dainippon Sumitomo Pharma, Mitsubishi Tanabe Pharma, Yoshitomi Yakuhin, Mochida Pharmaceutical, Otsuka Pharmaceutical, Kyowa Pharmaceutical, and Takeda Pharmaceutical. Dr. Suzuki has received manuscript or speaker's fees from Astellas, Dainippon Sumitomo Pharma, Eli Lilly, Elsevier Japan, Janssen Pharmaceuticals, Kyowa Yakuhin, Meiji Seika Pharma, Mitsubishi Tanabe Pharma, MSD, Novartis, Otsuka Pharmaceutical, Shionogi, Shire, Tsumura, Wiley Japan, and Yoshitomi Yakuhin, and research grants from Eisai, Mochida Pharmaceutical, Meiji Seika Pharma, and Shionogi. Dr. Azegawa has received speaker's honoraria from Eli Lilly, Mochida Pharmaceutical Co, Ltd, Otsuka Pharmaceutical, and Pfizer. Dr. Ueda has received manuscript fees or speaker's honoraria from Eli Lilly, Eisai Co. Ltd., Janssen Pharmaceutical, Meiji Seika Pharma, Mitsubishi Tanabe 
Pharma, MSD, Otsuka Pharmaceutical, Pfizer, Shionogi \& Co Ltd., Sumitomo Dainippon Pharma, Takeda Pharmaceutical, and Yoshitomi Yakuhin. Dr. Edagawa has received speaker's honoraria from Eli Lilly, Janssen Pharmaceutical K.K., Meiji Seika Pharma, Mitsubishi Tanabe Pharma, MSD, Otsuka Pharmaceutical, Pfizer, Sumitomo Dainippon Pharma, Kyowa and Yoshitomi Yakuhin. Dr. Katsumoto has received speaker's honoraria from Daiichi Sankyo, Eisai, Eli Lilly, Janssen Pharmaceutical, Kyowa Pharmaceutical, Meiji Seika Pharma, Mitsubishi Tanabe Pharma, MSD, Otsuka Pharmaceutical, Pfizer, Sumitomo Dainippon Pharma, Takeda Pharmaceutical Company Ltd., UCB Japan Co. Ltd. Dr. Kubota has received consultant fees from Pfizer and MeijiSeika Pharma and speaker's honoraria from Meiji-Seika Pharma, Eli Lilly, Janssen Pharmaceutical, Dainippon Sumitomo Pharma, Mitsubishi Tanabe Pharma, Yoshitomi Yakuhin, Otsuka Pharmaceutical, Takeda Pharmaceutical Company Ltd., and Eisai. Dr. Goto has received manuscript fees or speaker's honoraria from Eisai, Eli Lilly, Janssen Pharmaceutical, Kyowa Pharmaceutical Industry, Meiji Seika Pharma, Mitsubishi Tanabe Pharma, MSD, Otsuka Pharmaceutical, Sumitomo Dainippon Pharma, Towa Pharmaceutical. Dr. Hongo has received manuscript fees or speaker's honoraria from Eli Lilly, Janssen Pharmaceutical, Kyowa Pharmaceutical, Meiji Seika Pharma, Mitsubishi Mochida Seiyaku, Takeda Yakuhin, Shionogi Seiyaku, Shire, Tanabe Pharma, Otsuka Pharmaceutical, Pfizer, Shionogi, Sumitomo Dainippon Pharma, and Yoshitomi Yakuhin. Dr. Kato has received grant funding from Japan Society for the Promotion of Science, SENSHIN Medical Research Foundation and Japan Research Foundation for Clinical Pharmacology, and speaker's honoraria from DainipponSumitomo Pharma, Otsuka, Meiji-Seika Pharma, Eli Lilly, MSD K.K., GlaxoSmithkline, Pfizer, Janssen Pharmaceutical, Shionogi, Mitsubishi Tanabe Pharma, Takeda Pharmaceutical and Ono Pharmaceutical. Dr. YasuiFurukori has received grant/research support or honoraria from, and been on the speakers of Dainippon-Sumitomo Pharma, Mochida Pharmaceutical, MSD, and Otsuka Pharmaceutical. Dr. Yoshimura has received speaker's honoraria from Eli Lilly, Janssen, Dainippon Sumitomo, Otsuka, Meiji, Pfizer and Shionogi. Dr. Nakagawa has received lecture fees from Pfizer, Eli Lilly, Otsuka, Janssen Pharmaceutical, Mitsubishi Tanabe, Mochida, Dainippon Sumitomo, Johnson \& Johnson, Takeda, and NTT Docomo, and participated in an advisory board for Takeda, Meiji Seika and Tsumura. Dr. Kikuchi has received consultant fees from Takeda
Pharmaceutical, Dainippon Sumitomo, Eli Lilly, MSD, Pfizer, Yoshitomi Yakuhin, Mochida, and Center for Cognitive Behavioral Therapy and Training. Dr. Watanabe has received manuscript fees or speaker's honoraria from Daiichi Sankyo, Eisai, Eli Lilly, GlaxoSmithKline, Janssen Pharmaceutical, Kyowa Pharmaceutical, Meiji Seika Pharma, Mitsubishi Tanabe Pharma, MSD, Otsuka Pharmaceutical, Pfizer, Shionogi, Sumitomo Dainippon Pharma, Takeda Pharmaceutical, Yoshitomi Yakuhin, and has received research/grant support from Astellas Pharma, Daiichi Sankyo, Eisai, MSD, Mitsubishi Tanabe Pharma, Meiji Seika Pharma, Otsuka Pharmaceutical, Pfizer, Shionogi, Sumitomo Dainippon Pharma, and is a consultant of Eisai, Eli Lilly, Kyowa Pharmaceutical, Otsuka Pharmaceutical, Pfizer, Sumitomo Dainippon Pharma, Taisho Toyama Pharmaceutical, and Takeda Pharmaceutical. The authors report no other conflicts of interest in this work.

\section{References}

1. Perlis RH, Delbello MP, Miyahara S, Wisniewski SR, Sachs GS, Nierenberg AA. Revisiting depressive-prone bipolar disorder: polarity of initial mood episode and disease course among bipolar I systematic treatment enhancement program for bipolar disorder participants. Biol Psychiatry. 2005;58(7):549-553. doi:10.1016/j.biopsych.2005.07.029

2. Yatham LN, Kennedy SH, Parikh SV, et al. Canadian Network for Mood and Anxiety Treatments (CANMAT) and International Society for Bipolar Disorders (ISBD) 2018 guidelines for the management of patients with bipolar disorder. Bipolar Disord. 2018;20(2):97-170.

3. Fountoulakis KN, Grunze H, Vieta E, et al. The International College of Neuro-Psychopharmacology (CINP) treatment guidelines for bipolar disorder in adults (CINP-BD-2017), Part 3: the clinical guidelines. Int $J$ Neuropsychopharmacol. 2017;20(2):180-195. doi:10.1093/ijnp/ pyw 109

4. Grunze H, Vieta E, Goodwin GM, et al. The World Federation of Societies of Biological Psychiatry (WFSBP) guidelines for the biological treatment of bipolar disorders: update 2009 on the treatment of acute mania. World $J$ Biol Psychiatry. 2009;10(2):85-116. doi:10.1080/15622970902823202

5. Yildiz A, Nikodem M, Vieta E, Correll CU, Baldessarini RJ. A network meta-analysis on comparative efficacy and all-cause discontinuation of antimanic treatments in acute bipolar mania. Psychol Med. 2015;45(2):299-317. doi:10.1017/S0033291714001305

6. Miura T, Noma H, Furukawa TA, et al. Comparative efficacy and tolerability of pharmacological treatments in the maintenance treatment of bipolar disorder: a systematic review and network meta-analysis. Lancet Psychiatry. 2014;1(5):351-359. doi:10.1016/ S2215-0366(14)70314-1

7. Taylor DM, Cornelius V, Smith L, Young AH. Comparative efficacy and acceptability of drug treatments for bipolar depression: a multiple-treatments meta-analysis. Acta Psychiatr Scand. 2014;130 (6):452-469. doi:10.1111/acps.2014.130.issue-6

8. Anderson IM, Haddad PM, Scott J. Bipolar disorder. BMJ. 2012;345: e8508. doi:10.1136/bmj.e8508

9. Sachs GS, Thase ME, Otto MW, et al. Rationale, design, and methods of the systematic treatment enhancement program for bipolar disorder (STEP-BD). Biol Psychiatry. 2003;53(11):1028-1042. doi:10.1016/ S0006-3223(03)00165-3 
10. Cipriani A, Barbui C, Rendell J, Geddes JR. Clinical and regulatory implications of active run-in phases in long-term studies for bipolar disorder. Acta Psychiatr Scand. 2014;129(5):328-342. doi:10.1111/ acps.2014.129.issue-5

11. Angst J, Azorin JM, Bowden CL, et al. Prevalence and characteristics of undiagnosed bipolar disorders in patients with a major depressive episode: the BRIDGE study. Arch Gen Psychiatry. 2011;68 (8):791-798. doi:10.1001/archgenpsychiatry.2011.87

12. Phillips ML, Kupfer DJ. Bipolar disorder diagnosis: challenges and future directions. Lancet. 2013;381(9878):1663-1671. doi:10.1016/ S0140-6736(13)60989-7

13. Carvalho AF, Takwoingi Y, Sales PMG, et al. Screening for bipolar spectrum disorders: a comprehensive meta-analysis of accuracy studies. J Affect Disord. 2015;172:337-346. doi:10.1016/j.jad. 2014.10.024

14. Muneer A. Staging models in bipolar disorder: a systematic review of the literature. Clin Psychopharmacol Neurosci. 2016;14(2):117-130. doi:10.9758/cpn.2016.14.2.117

15. Tohen M, Zarate CA Jr., Hennen J, et al. The McLean-Harvard first-episode mania study: prediction of recovery and first recurrence. Am J Psychiatry. 2003;160(12):2099-2107. doi:10.1176/ appi.ajp.160.12.2099

16. Haro JM, Reed C, Gonzalez-Pinto A, Novick D, Bertsch J, Vieta E. 2-Year course of bipolar disorder type I patients in outpatient care: factors associated with remission and functional recovery. Eur Neuropsychopharmacol. 2011;21(4):287-293. doi:10.1016/j. euroneuro.2010.08.001

17. Tohen M, Frank E, Bowden CL, et al. The International Society for Bipolar Disorders (ISBD) task force report on the nomenclature of course and outcome in bipolar disorders. Bipolar Disord. 2009;11 (5):453-473. doi:10.1111/bdi.2009.11.issue-5

18. Kogan JN, Otto MW, Bauer MS, et al. Demographic and diagnostic characteristics of the first 1000 patients enrolled in the Systematic Treatment Enhancement Program for Bipolar Disorder (STEP-BD). Bipolar Disord. 2004;6(6):460-469. doi:10.1111/bdi.2004.6.issue-6

19. MacCabe JH, Lambe MP, Cnattingius S, et al. Excellent school performance at age 16 and risk of adult bipolar disorder: national cohort study. Br J Psychiatry. 2010;196(2):109-115. doi:10.1192/bjp. bp. 108.060368

20. Kessler RC, Adler L, Barkley R, et al. The prevalence and correlates of adult ADHD in the United States: results from the national comorbidity survey replication. Am J Psychiatry. 2006;163 (4):716-723. doi:10.1176/ajp.2006.163.4.716

21. Tse S, Chan S, Ng KL, Yatham LN. Meta-analysis of predictors of favorable employment outcomes among individuals with bipolar disorder. Bipolar Disord. 2014;16(3):217-229. doi:10.1111/ bdi.2014.16.issue-3

22. Judd LL, Akiskal HS, Schettler PJ, et al. The long-term natural history of the weekly symptomatic status of bipolar I disorder. Arch Gen Psychiatry. 2002;59(6):530-537. doi:10.1001/archpsyc.59.6.530

23. Judd LL, Akiskal HS, Schettler PJ, et al. A prospective investigation of the natural history of the long-term weekly symptomatic status of bipolar II disorder. Arch Gen Psychiatry. 2003;60(3):261-269. doi:10.1001/archpsyc.60.3.261

Neuropsychiatric Disease and Treatment

\section{Publish your work in this journal}

Neuropsychiatric Disease and Treatment is an international, peerreviewed journal of clinical therapeutics and pharmacology focusing on concise rapid reporting of clinical or pre-clinical studies on a range of neuropsychiatric and neurological disorders. This journal is indexed on PubMed Central, the 'PsycINFO' database and CAS, and
24. Kanba S, Kato T, Terao T, Yamada K. Guideline for treatment of bipolar disorder by the Japanese society of mood disorders, 2012. Psychiatry Clin Neurosci. 2013;67(5):285-300. doi:10.1111/ pen. 12060

25. Sani G, Perugi G, Tondo L. Treatment of bipolar disorder in a lifetime perspective: is lithium still the best choice? Clin Drug Investig. 2017;37(8):713-727.

26. Sani G, Fiorillo A. The use of lithium in mixed states. CNS Spectr. 2019;1-3. doi:10.1017/S1092852919001184

27. Goodwin GM, Haddad PM, Ferrier IN, et al. Evidence-based guidelines for treating bipolar disorder: revised third edition recommendations from the British Association for Psychopharmacology. J Psychopharmacol. 2016;30(6):495-553. doi:10.1177/02698811 16636545

28. Li DJ, Lin CH, Chen FC. Factors affecting time to remission for inpatients with bipolar mania - A naturalistic Taiwanese study. J Affect Disord. 2018;232:73-78. doi:10.1016/j.jad.2018.02.032

29. Bukh JD, Andersen PK, Kessing LV. Rates and predictors of remission, recurrence and conversion to bipolar disorder after the first lifetime episode of depression-a prospective 5-year follow-up study. Psychol Med. 2016;46(6):1151-1161. doi:10.1017/S003329171 5002676

30. Farren CK, Hill KP, Weiss RD. Bipolar disorder and alcohol use disorder: a review. Curr Psychiatry Rep. 2012;14(6):659-666. doi:10.1007/s11920-012-0320-9

31. Ferrari AJ, Baxter AJ, Whiteford HA. A systematic review of the global distribution and availability of prevalence data for bipolar disorder. J Affect Disord. 2011;134(1-3):1-13. doi:10.1016/j.jad. 2010.11.007

32. Swann AC, Bowden CL, Calabrese JR, Dilsaver SC, Morris DD. Mania: differential effects of previous depressive and manic episodes on response to treatment. Acta Psychiatr Scand. 2000;101 (6):444-451. doi:10.1034/j.1600-0447.2000.101006444.x

33. Dawson EL, Shear PK, Howe SR, et al. Impulsivity predicts time to reach euthymia in adults with bipolar disorder. Bipolar Disord. 2014;16(8):846-856. doi:10.1111/bdi.2014.16.issue-8

34. Kemp DE, Ganocy SJ, Brecher M, et al. Clinical value of early partial symptomatic improvement in the prediction of response and remission during short-term treatment trials in 3369 subjects with bipolar I or II depression. J Affect Disord. 2011;130(1-2):171-179. doi:10.1016/j.jad.2010.10.026

35. Kemp DE, Johnson E, Wang WV, Tohen M, Calabrese JR. Clinical utility of early improvement to predict response or remission in acute mania: focus on olanzapine and risperidone. $J$ Clin Psychiatry. 2011;72(9):1236-1241. doi:10.4088/JCP.09m05874yel

36. Perugi G, Medda P, Barbuti M, Novi M, Tripodi B. The role of electroconvulsive therapy in the treatment of severe bipolar mixed state. Psychiatr Clin North Am. 2020;43(1):187-197. doi:10.1016/j. psc.2019.10.010

37. Bersani FS, Girardi N, Sanna L, et al. Deep transcranial magnetic stimulation for treatment-resistant bipolar depression: a case report of acute and maintenance efficacy. Neurocase. 2013;19(5):451-457. doi:10.1080/13554794.2012.690429

\section{Dovepress}

is the official journal of The International Neuropsychiatric Association (INA). The manuscript management system is completely online and includes a very quick and fair peer-review system, which is all easy to use. Visit http://www.dovepress.com/testimonials.php to read real quotes from published authors. 